\title{
ORTHOGONAL POLYNOMIALS ON THE UNIT CIRCLE WITH VERBLUNSKY COEFFICIENTS DEFINED BY THE SKEW-SHIFT
}

\author{
HELGE KRÜGER
}

\begin{abstract}
I give an example of a family of orthogonal polynomials on the unit circle with Verblunsky coefficients given by the skew-shift for which the associated measures are supported on the entire unit circle and almost-every Aleksandrov measure is pure point.

Furthermore, I show in the case of the two dimensional skew-shift the zeros of para-orthogonal polynomials obey the same statistics as an appropriate irrational rotation.

The proof is based on an analysis of the associated CMV matrices.
\end{abstract}

\section{INTRODUCTION}

In this article, I consider orthogonal polynomials on the unit circle, whose Verblunsky coefficients are given by

$$
\alpha_{n}=\lambda \mathrm{e}^{2 \pi \mathrm{i} \cdot \omega n^{k}}
$$

for $0 \neq \lambda \in \mathbb{D}=\{z:|z|<1\}, \omega$ an irrational number, and $k \geq 2$. The case $k=1$ corresponds to rotated versions of the Geronimus polynomials, see Theorem 1.6.13 in [30] and Proposition 2.3 (see also Theorem 5.3 in [16]). Given Verblunsky coefficients $\alpha_{n}$, we define orthogonal polynomials on the unit circle recursively by

$$
\Phi_{0}(z)=1, \quad \Phi_{n+1}(z)=z \Phi_{n}(z)-\overline{\alpha_{n}} \Phi_{n}^{*}(z),
$$

where $\Phi_{n}^{*}(z)=z^{n} \overline{\Phi\left(\bar{z}^{-1}\right)}$ is the reversed polynomial. By Verblunsky's theorem, there exists an unique probability measure $\mu$ on $\partial \mathbb{D}$ such that the $\Phi_{n}$ are orthogonal with respect to it. The first result is

Theorem 1.1. The support of $\mu$ satisfies

$$
\operatorname{supp}(\mu)=\partial \mathbb{D} \text {. }
$$

The key to the proof of this theorem is that the support of $\mu$ is the same as the support of the measure with Verblunsky coefficients $\alpha_{n} \mathrm{e}^{2 \pi \mathrm{i} y n}$ by ergodicity for any $y \in \mathbb{T}=\mathbb{R} / \mathbb{Z}$. Now these two supports are just rotated versions of each other. Hence $\operatorname{supp}(\mu)$ must be the entire unit circle. I give the details of the proof in Section 2 ,

Next, consider the family of Verblunsky coefficients given by $\alpha_{x, n}=\alpha_{n} \cdot \mathrm{e}^{2 \pi \mathrm{i} x}$. The corresponding measures are known as Aleksandrov measures $\mu_{x}$ see Section 3.2. in [29. Then we have that

Date: November 18, 2011.

Key words and phrases. OPUC, CMV matrices, spectrum, skew-shift, eigenvalue statistics.

H. K. was supported by a fellowship of the Simons foundation. 
Theorem 1.2. For almost every $x$, the Aleksandrov measure $\mu_{x}$ is pure point.

The proof of this theorem is essentially the same as Theorem 1.1. since the rotational invariance implies positivity of the Lyapunov exponent. Pure point spectrum then follows from spectral averaging. Deterministic examples with similar properties have been previously obtained in 13 .

Adapting the methods of [21, 22] to orthogonal polynomials on the unit circle, it should be possible to obtain similar even for $k>1$ not an integer.

At this point, let me mention that the corresponding question for orthogonal polynomials on the real line respectively better Schrödinger operators is open. Consider the potential $V(n)=\lambda \cos \left(2 \pi \omega n^{2}\right)$ for an irrational number $\omega$. Then under a Diophantine assumption on $\omega$ and a largeness condition on $\lambda$ one can show pure point spectrum, see [9], [10], and Chapter 15 in [5] and that the spectrum contains intervals 24]. However, it is believed that for all $\lambda>0$ the spectrum of this operator is an interval and pure point. Partial results for $\lambda>0$ small can be found in 6, 7, 8].

The proofs of Theorem 1.1 and 1.2 are much easier than the real case, because of algebraic miracles (Proposition 5.1). However, there is also an analytic reason why the case on the unit circle should be simpler, namely that then the spectrum has no edges.

For this reason, I expect it to be possible to show analogs of Theorem 1.1 and 1.2 if one perturbs $\alpha_{n}$ slightly by for example $\alpha_{n}+\varepsilon f\left(\omega n^{k}\right)$ for an analytic and one-periodic function $f$ and $\varepsilon>0$ small enough.

At first sight Theorem 1.1 and 1.2 might not seem too surprising, since we know many measures whose support is the entire unit circle. But the Verblunsky coefficients of these measures behave quite differently, for regular measures one knows [28] that the Verblunsky coefficients Cesáro sum to 0. Similarly non-zero periodic potentials have at least one gap.

The situation becomes even more striking when considering Schrödinger operators. There have been a series of innovative works [1, 2, 3, 4, 17, 18, to prove Cantor spectrum, whereas there are only the perturbative methods from [12, 24] to prove that the spectrum contains an interval.

Finally, I also want to address the zero distribution of the para-orthogonal polynomials. This question has not been discussed for Schrödinger operators yet. Define for $\beta \in \partial \mathbb{D}$

$$
\Phi_{n}(z ; \beta)=z \Phi_{n-1}(z)-\bar{\beta} \Phi_{n-1}^{*}(z) .
$$

In difference to $\Phi_{n}(z)$ the zeros of $\Phi_{n}(z ; \beta)$ are on the unit circle. Denote these zeros by $\mathrm{e}^{2 \pi \mathrm{i} \theta_{1}}, \ldots, \mathrm{e}^{2 \pi \mathrm{i} \theta_{N}}$. An inspection of the proof of Theorem 6.1 shows that an appropriate adaption of the results would remain true for $\Phi_{n}(z)$.

Before stating our main result, I will now illustrate the behavior of the zeros with some numerical computations. Order the values $\theta_{j}$ such that

$$
0 \leq \theta_{1}<\theta_{2}<\cdots<\theta_{N}<1 .
$$

Define the length of gaps by

$$
g_{j}=\theta_{j+1}-\theta_{j} .
$$




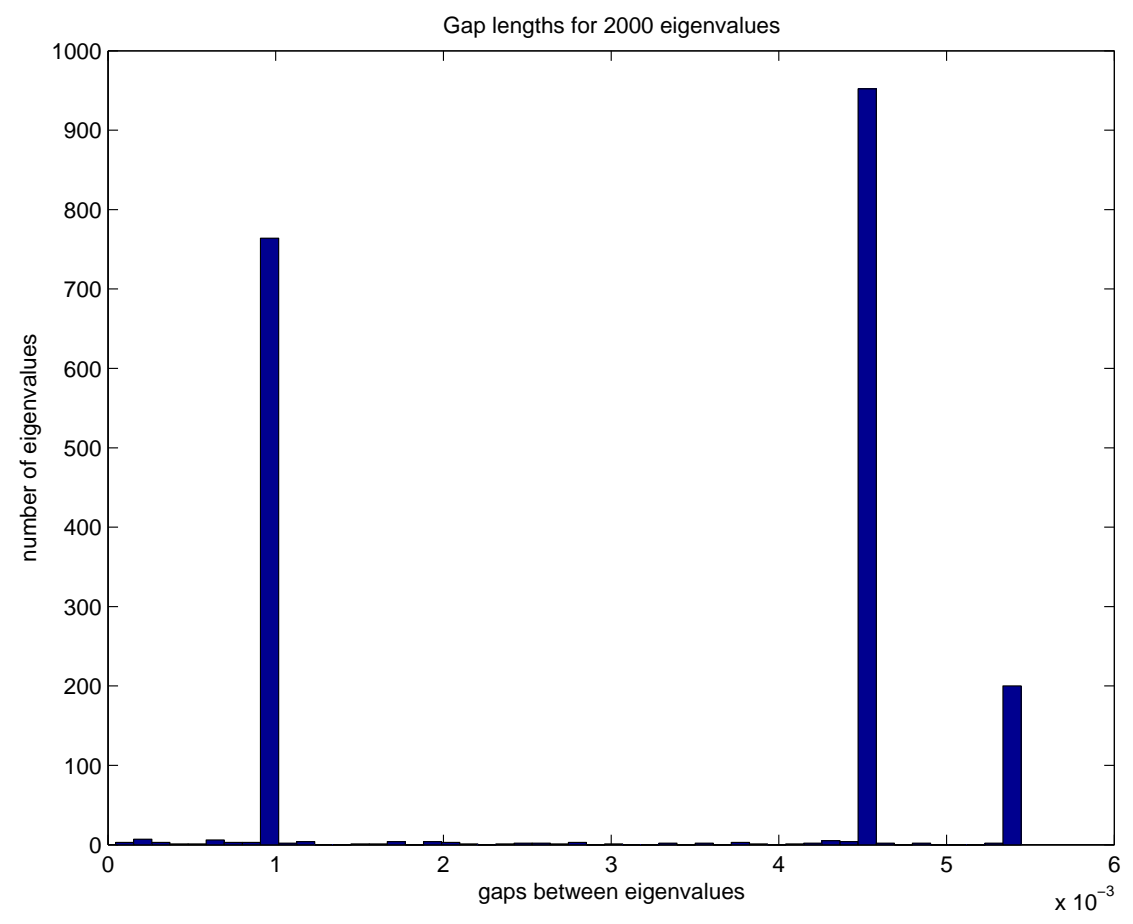

Figure 1. Zeros of $\Phi_{2000}(z ; \beta)$ for $k=2$ and $\omega=\sqrt{2}$.

Figure 1 and 2 show the distribution of the values of $g_{j}$ for different values of $N$ when $k=2$. One sees that this distribution peaks at only three values. This should remind one of the distribution of gap lengths for the sequence of values $\{\eta n$ $(\bmod 1)\}_{n=1}^{N}$ for some value of $\eta$ and in fact, we will show this in Theorem 1.3 Also it should be pointed out that these gap distributions do not converge.

On the other hand Figure 3 shows the same graphic for $k=3$ and the distribution resembles an exponential distribution. One obtains similar figures for $k \geq 4$. This is the same distribution one would obtain if the $\theta_{j}$ were given by a Poisson process and by [34] also if the the Verblunsky coefficients $\alpha_{n}$ were given by independent identically distributed random variables whose distribution is non constant and rotationally invariant.

Finally, in the case $k=1$, the (rotated) Geronimus Polynomials, the assumptions of the Freud-Levin theorem hold (Theorem 2.6.10 in [33]) and one has clock spacing, so the spacing is given by the inverse of the corresponding density of states measure. This measure turns out to be non-constant, so there is not a single peak.

In order to state our result, we need to introduce more notation. Define the Laplace functional of $N$ points $x_{1}, \ldots, x_{N} \in \mathbb{T}$ by

$$
\mathfrak{L}_{\underline{x}, N}(f)=\int_{\mathbb{T}} \exp \left(-\sum_{n=1}^{N} f\left(N x_{n}(\theta)\right)\right) d \theta,
$$

where $\left[-\frac{1}{2}, \frac{1}{2}\right) \ni x_{n}(\theta)=x_{n}-\theta(\bmod 1)$ and $f \geq 0$ is continuous and compactly supported function. See [20] for a discussion of Laplace functionals related to zeros of paraorthogonal polynomials. 


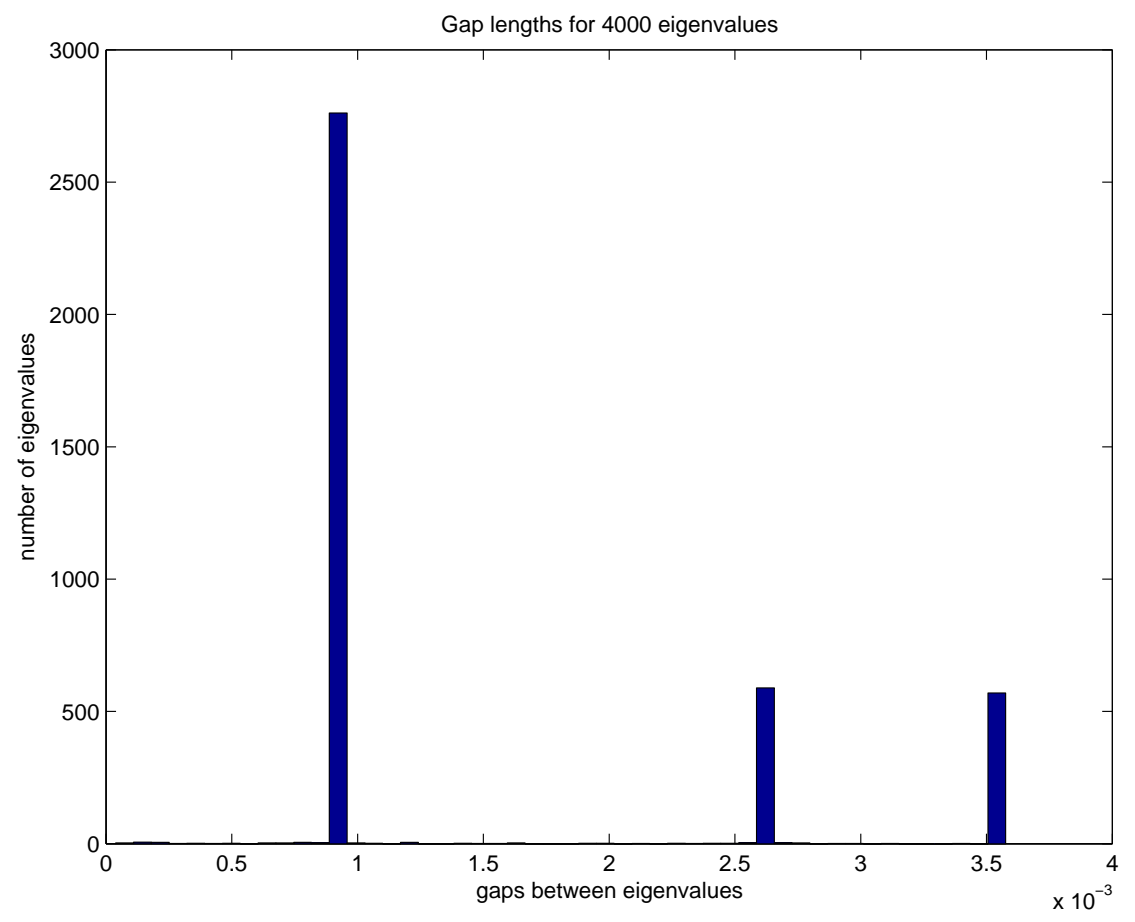

Figure 2. Zeros of $\Phi_{4000}(z ; \beta)$ for $k=2$ and $\omega=\sqrt{2}$.

Denote by $\mathfrak{L}_{\omega, N}^{R}$ the Laplace functional of the sequence of points $\{n \omega(\bmod 1)\}_{n=1}^{N}$. The behavior of this sequence is well understood, see for example [27. In particular, this quantity does not converge to a limit. We will show

Theorem 1.3. Let $k=2, \tau>1$ and assume that $\omega$ satisfies

$$
\inf _{q \geq 1, q \in \mathbb{Z}} q^{\tau} \operatorname{dist}(q \omega, \mathbb{Z})>0 .
$$

Then for any positive, continuous, and compactly supported function $f: \mathbb{R} \rightarrow \mathbb{R}$, we have

$$
\lim _{N \rightarrow \infty}\left(\mathfrak{L}_{\underline{\theta}, N}(f)-\mathfrak{L}_{2 \omega, N}^{R}(f)\right)=0 .
$$

This says that the values of $\mathfrak{L}_{\underline{\theta}, N}$ are deterministic in the large $N$ limit. However, they do not converge to a single value as the one for the irrational rotation does not. Using either Theorem 1.3 or easier Theorem 6.1. one can show that the gap distribution of the eigenvalues indeed obeys the distribution shown in Figure 1 and 2 The Diophantine assumption (1.8) is necessary, I sketch an argument in Remark 1.4 Furthermore, it should be noted that Lebesgue almost every $\omega$ satisfies (1.8).

In this sense the case $k=2$ is of intermediate disorder, one has pure point spectrum with exponentially decaying eigenfunctions, but one does not have sufficient independence to obtain Poisson statistics.

The definition of the Laplace functional given here is different from the one usually given in the theory of point processes. There, one does not introduce averaging 


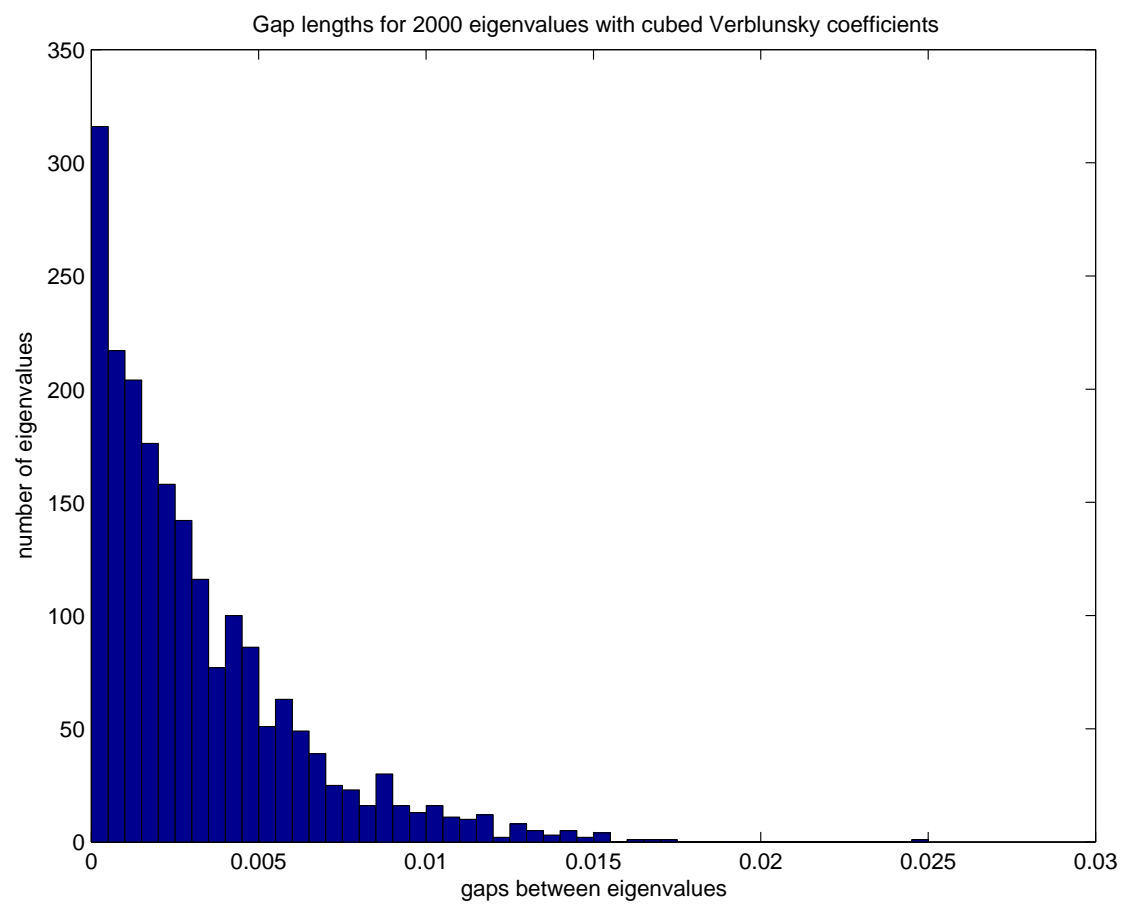

Figure 3. Zeros of $\Phi_{4000}(z ; \beta)$ for $k=2$ and $\omega=\sqrt{2}$.

over the unit circle by hand, but this comes from the points $x_{n}$ being defined on some probability space. In Section 5, we will see that our Verblunsky coefficients are defined on a probability space, and that averaging over it in particular contains the $\theta$ average. Hence, the name Laplace functional is justified.

Remark 1.4. Assume that for coprime integers $p, q, N$ very large, and $\delta>0 a$ small parameter, we have that $\left|\omega-\frac{p}{q}\right| \leq \frac{1}{N^{3+\delta}}$. Then for $1 \leq n \leq N$, we have that

$$
\left|\alpha_{n}-\lambda \mathrm{e}^{2 \pi \mathrm{i} \frac{p n^{2}}{q}}\right| \leq \frac{1}{N^{1+\frac{\delta}{2}}} .
$$

Since the Verblunsky coefficients $\lambda \mathrm{e}^{2 \pi \mathrm{i} \frac{p n^{2}}{q}}$ are q-periodic, the corresponding zeros of the paraorthogonal polynomials are clock-spaced, so of size $\frac{1}{N}$, whereas the points $\{2 n \omega(\bmod 1)\}_{n=1}^{N}$ are all in a $\frac{1}{N^{2+\delta}}$ neighborhood of the points $\left\{\frac{\ell}{q}\right\}_{\ell=1}^{q}$.

These two behaviors are clearly incompatible, and thus Theorem 1.3 cannot hold for Liouville frequencies.

Let me now outline the rest of the content of the paper. Section 2 discusses the basic theory of half-line CMV matrices and gives the proof of Theorem 1.1 Then Section 3 introduces extended CMV operators, so ones defined on the whole-line, discusses restrictions of these, defines the Green's function, and derives useful formulas relating determinants of CMV matrices to transfer matrices. This discussion is somewhat more complicated than the case of Schrödinger operators. Section 4 
combines the formulas from the previous section with the ones for ergodic CMV matrices. In Section 5 CMV matrices with built-in rotational invariance are discussed and Theorem 1.2 is proven.

In Section 6, we prove Theorem 1.3 relying on results from Sections 7 and 8 Basically, Section 8 improves the bounds on decay of the Green's function obtained in Section 4 from unique ergodicity by using quantitative recurrence results for the skew-shift discussed in Appendix [A. Section 7 shows how to exploit Section 8 to obtain good test functions.

\section{A first LOOK at the CMV matrix}

In this section, we take a look at half-line CMV matrices and provide a proof of Theorem 1.1. In the following sections, we will discuss whole line CMV matrices in more details. Although most results in this section will be reproven in later parts, I have included it, since it is closed to the notation of [29, 30.

Let $\left\{\alpha_{n}\right\}_{n=0}^{\infty}$ be a sequence of Verblunsky coefficients. Define $\rho_{n}=\left(1-\left|\alpha_{n}\right|^{2}\right)^{\frac{1}{2}}$ and the unitary matrices

$$
\Theta_{n}=\left(\begin{array}{cc}
\overline{\alpha_{n}} & \rho_{n} \\
\rho_{n} & -\alpha_{n}
\end{array}\right)
$$

Define the operators $\mathcal{L}_{+}, \mathcal{M}_{+}$by

$$
\mathcal{L}_{+}=\left(\begin{array}{ccc}
\Theta_{0} & & \\
& \Theta_{2} & \\
& & \ddots
\end{array}\right), \quad \mathcal{M}_{+}=\left(\begin{array}{ccc}
1 & & \\
& \Theta_{1} & \\
& & \ddots
\end{array}\right)
$$

where 1 represents the identity $1 \times 1$ matrix. The CMV matrix is then defined by $\mathcal{C}=\mathcal{L}_{+} \mathcal{M}_{+}$which will be five-diagonal and unitary. Its importance comes from that the measure $\mu$ associated to the Verblunsky coefficients $\left\{\alpha_{n}\right\}_{n=0}^{\infty}$ is the spectral measure of $\delta_{0}$ with respect to $\mathcal{C}$, so one has

$$
\int_{\partial \mathbb{D}} z^{n} d \mu(z)=\left\langle\delta_{0}, \mathcal{C}^{n} \delta_{0}\right\rangle
$$

We denote by $\operatorname{supp}_{\mathrm{ess}}(\mu)$ the essential support of the measure $\mu$, that is the support of $\mu$ with point masses removed.

Lemma 2.1. Define $\tilde{\alpha}_{n}=\alpha_{n+1}$. Let $\tilde{\mu}$ be the measure corresponding to $\left\{\tilde{\alpha}_{n}\right\}_{n=0}^{\infty}$. Then

$$
\operatorname{supp}_{\mathrm{ess}}(\tilde{\mu})=\operatorname{supp}_{\mathrm{ess}}(\mu)
$$

Proof. Clearly $\operatorname{supp}_{\mathrm{ess}}(\mu)=\sigma_{\mathrm{ess}}(\mathcal{C})$. Let $S$ be the backward shift on $\ell^{2}(\mathbb{N})$. Then $\mathcal{C}$ and $S^{*} \tilde{\mathcal{C}} S$ differ by a finite rank operator. The claim follows.

A similar proof implies that for all the translates $\alpha_{n}^{\ell}=\alpha_{n+\ell}$ the corresponding CMV matrices have the same essential spectrum. Hence, for Verblunsky coefficients given by (1.1), one obtains that the family of Verblunsky coefficients given by

$$
\alpha_{n}^{\ell}=\lambda \exp \left(2 \pi \mathrm{i}\left(\omega n^{k}+\sum_{j=0}^{k-1}\left(\begin{array}{l}
k \\
j
\end{array}\right) \omega \ell^{k-j} \cdot n^{j}\right)\right)
$$


have the same essential spectrum. Define for $y \in[0,1]^{k}$ a family of Verblunsky coefficients by

$$
\tilde{\alpha}_{y, n}=\lambda \exp \left(2 \pi \mathrm{i}\left(\omega n^{k}+\sum_{j=0}^{k-1} y_{j} \cdot n^{j}\right)\right) .
$$

Lemma 2.2. We have for any $y \in[0,1]^{k}$ that

$$
\sigma_{\mathrm{ess}}(\mathcal{C})=\sigma_{\mathrm{ess}}\left(\widetilde{\mathcal{C}}_{y}\right)
$$

Proof. Given $y$, there exists a sequence $\ell_{s}$ such that

$$
\left(\begin{array}{c}
k \\
j
\end{array}\right) \omega \ell_{s}^{k-j} \rightarrow y_{j}
$$

for $0 \leq j \leq k-1$ as $s \rightarrow \infty$ (see Theorem 2.2 and Lemma 2.3 in [21]). By strong convergence, one thus obtains that

$$
\sigma_{\text {ess }}(\mathcal{C}) \supseteq \sigma_{\text {ess }}\left(\widetilde{\mathcal{C}}_{y}\right) .
$$

The other inclusion can be proven in a similar way.

Results similar to Lemma 2.2 have been discussed in [25]. For the proof of Theorem 1.1, we will also need

Proposition 2.3. Define Verblunsky coefficients by $\tilde{\alpha}_{n}=\mathrm{e}^{2 \pi \mathrm{i} \eta n} \alpha_{n}$. Then

$$
\operatorname{supp}(\tilde{\mu})=\mathrm{e}^{-2 \pi \mathrm{i} \eta} \operatorname{supp}(\mu) .
$$

Proof. This follows from the formulas in Appendix A.H. in 30. I will also give another proof in Section 5 ,

Given $y \in[0,1]^{k}$ and $\eta \in[0,1]$ define

$$
\hat{y}_{j}= \begin{cases}y_{j}, & j=0,2 \leq j \leq k-1 ; \\ y_{1}+\eta, & j=1 .\end{cases}
$$

Proposition 2.3 shows that

$$
\sigma_{\text {ess }}\left(\widetilde{\mathcal{C}_{y}}\right)=\mathrm{e}^{-2 \pi \mathrm{i} \eta} \sigma_{\text {ess }}\left(\widetilde{\mathcal{C}}_{\hat{y}}\right) .
$$

Having this, we are now ready for

Proof of Theorem 1.1. The results discussed so far imply that $\sigma_{\text {ess }}(\mathcal{C})$ is a nonempty, rotationally invariant, subset of $\partial \mathbb{D}$. Hence, we must have

$$
\sigma_{\mathrm{ess}}(\mathcal{C})=\partial \mathbb{D}
$$

Since also $\sigma_{\text {ess }}(\mathcal{C}) \subseteq \sigma(\mathcal{C}) \subseteq \partial \mathbb{D}$, the claim follows. 


\section{EXTENDED CMV OPERATORS}

In this section, we introduce extended CMV operators and discuss their properties that will be useful to us. See also [15] and Section 10.5 in [30] for discussions from different viewpoints.

Let now $\left\{\alpha_{n}\right\}_{n \in \mathbb{Z}}$ be a bi-infinite sequence of Verblunsky coefficients, i.e. $\alpha_{n} \in \mathbb{D}$ although we will discuss setting certain $\alpha_{n}$ to values in $\overline{\mathbb{D}}$ below. Recall that $\rho_{n}=\left(1-\left|\alpha_{n}\right|^{2}\right)^{\frac{1}{2}}$ and

$$
\Theta_{n}=\left(\begin{array}{cc}
\overline{\alpha_{n}} & \rho_{n} \\
\rho_{n} & -\alpha_{n}
\end{array}\right)
$$

viewed as acting on $\ell^{2}(\{n, n+1\})$. Define

$$
\mathcal{L}=\bigoplus_{n \text { even }} \Theta_{n}, \quad \mathcal{M}=\bigoplus_{n \text { odd }} \Theta_{n}
$$

and the extended CMV operator $\mathcal{E}=\mathcal{L} \cdot \mathcal{M}$. We note

Lemma 3.1. $\mathcal{E}, \mathcal{L}$, and $\mathcal{M}$ are unitary operators $\ell^{2}(\mathbb{Z}) \rightarrow \ell^{2}(\mathbb{Z})$. Furthermore, $\mathcal{L}$ leaves the subspaces $\ell^{2}(\{n, n+1\})$ for $n$ even invariant, whereas $\mathcal{L}$ does this for $n$ odd.

We will now discuss various restrictions of CMV operators. First denote by $P^{[a, b]}$ the projection $\ell^{2}(\mathbb{Z}) \rightarrow \ell^{2}([a, b])$. We define

$$
X^{[a, b]}=\left(P^{[a, b]}\right)^{*} X P^{[a, b]}
$$

for $X \in\{\mathcal{E}, \mathcal{M}, \mathcal{L}\}$.

Lemma 3.2. $\mathcal{E}^{[a, b]}=\mathcal{L}^{[a, b]} \mathcal{M}^{[a, b]}$.

Proof. Compute.

It is easy to check that the operator $\mathcal{E}^{[a, b]}$ will no longer be unitary, but it will still be an useful object. Let now $\beta \in \partial \mathbb{D}$ and $a \in \mathbb{Z}$ and consider the modified Verblunsky coefficients

$$
\tilde{\alpha}_{n}= \begin{cases}\alpha_{n}, & n \neq a \\ \beta, & n=a .\end{cases}
$$

We then have that $\widetilde{\mathcal{E}}, \widetilde{\mathcal{L}}$, and $\widetilde{\mathcal{M}}$ leave the spaces $\ell^{2}(\{a+1, a+2, \ldots\})$ and $\ell^{2}(\{\ldots, a-$ $1, a\})$ invariant. In particular, we can define unitary restrictions

$$
\mathcal{E}_{\beta, \bullet}^{[a+1, \infty)}=P^{[a+1, \infty)} \widetilde{\mathcal{E}} P^{[a+1, \infty)}, \quad \mathcal{E}_{\bullet, \beta}^{(-\infty, a]}=P^{(-\infty, a]} \widetilde{\mathcal{E}} P^{(-\infty, a]} .
$$

Lemma 3.3. Let $\mathcal{C}$ be the $C M V$ operator with Verblunksy coefficients $\left\{\alpha_{n}\right\}_{n=0}^{\infty}$. Then

$$
\mathcal{C}=\mathcal{E}_{1, \bullet}^{[0, \infty)} .
$$

Denote by $R$ the identification $\ell^{2}(\{\ldots,-2,-1\})$ with $\ell^{2}(\{0,1,2, \ldots\})$ and by $\mathcal{C}_{-}$ the CMV operator with Verblunsky coefficients $\left\{-\bar{\alpha}_{-n-1}\right\}_{n=0}^{\infty}$. Then

$$
\mathcal{C}_{-}=R \mathcal{E}_{\bullet, 1}^{(-\infty,-1]} R^{*} .
$$

Proof. These are computations. 
We will now consider restrictions to intervals. So let $a<b$ be integers, and $\beta, \gamma \in \partial \mathbb{D}$. Define a sequence of Verblunsky coefficients

$$
\tilde{\alpha}_{n}= \begin{cases}\beta, & n=a \\ \gamma, & n=b ; \\ \alpha_{n}, & n \notin\{a, b\}\end{cases}
$$

We then define the operator

$$
\mathcal{E}_{\beta, \gamma}^{[a+1, b]}=P^{[a+1, b]} \widetilde{\mathcal{E}} P^{[a+1, b]} .
$$

Of course, this definition makes sense for $\beta, \gamma \in \overline{\mathbb{D}}$ and $a=-\infty$ or $b=\infty$. Furthermore, we write $\bullet$ if we leave $\alpha_{a}$ or $\alpha_{b}$ unchanged to match the previous definition. $\beta, \gamma \in \partial \mathbb{D}$ should be thought of as boundary conditions.

Lemma 3.4. If $\beta, \gamma \in \partial \mathbb{D}$ then $\mathcal{E}_{\beta, \gamma}^{[a, b]}, \mathcal{L}_{\beta, \gamma}^{[a, b]}$, and $\mathcal{M}_{\beta, \gamma}^{[a, b]}$ are unitary.

Since the equation $\mathcal{E} \psi=z \psi$ is equivalent to $\left(z \mathcal{L}^{*}-\mathcal{M}\right) \psi=0$. We note for further reference

Lemma 3.5. The matrix $A=z\left(\mathcal{L}_{\beta, \gamma}^{[a, b]}\right)^{*}-\mathcal{M}_{\beta, \gamma}^{[a, b]}$ is tridiagonal. Write $A=$ $\left\{A_{i, j}\right\}_{a \leq i, j \leq b}$. Then we have that

$$
A_{j, j}=\left\{\begin{array}{ll}
z \alpha_{j}+\alpha_{j-1}, & j \text { even } \\
-z \overline{\alpha_{j-1}}-\overline{\alpha_{j}}, & j \text { odd },
\end{array} \quad A_{j+1, j}=A_{j, j+1}=\tilde{\rho}_{j}= \begin{cases}z \rho_{j}, & j \text { even }, \\
-\rho_{j}, & j \text { odd } .\end{cases}\right.
$$

Let $z \in \mathbb{C}, \beta, \gamma \in \partial D, a \leq k, \ell \leq b$, then the Green's function is defined by

$$
G_{\beta, \gamma}^{[a, b]}(z ; k, \ell)=\left\langle\delta_{k},\left(z\left(\mathcal{L}_{\beta, \gamma}^{[a, b]}\right)^{*}-\mathcal{M}_{\beta, \gamma}^{[a, b]}\right)^{-1} \delta_{\ell}\right\rangle .
$$

Our goal now will be to provide a formula for the Green's function in terms of quantities that are easier to analyze, like the formula for the Green's function of Schrödinger operators in term of orthogonal polynomials, respectively entries of the transfer matrix.

We define

$$
\begin{aligned}
\Phi_{\beta, \gamma}^{[a, b]}(z) & =\operatorname{det}\left(z-\mathcal{E}_{\beta, \gamma}^{[a, b]}\right) \\
& =\operatorname{det}\left(z\left(\mathcal{L}_{\beta, \gamma}^{[a, b]}\right)^{*}-\mathcal{M}_{\beta, \gamma}^{[a, b]}\right) \cdot \operatorname{det}\left(\left(\mathcal{L}_{\beta, \gamma}^{[a, b]}\right)^{*}\right)
\end{aligned}
$$

and

$$
\varphi_{\beta, \gamma}^{[a, b]}(z)=\left(\rho_{a} \cdots \rho_{b}\right)^{-1} \Phi_{\beta, \gamma}^{[a, b]}(z) .
$$

Lemma 3.6. Let $\Phi_{n}(z)$ be defined as in (1.2). Then

$$
\Phi_{n}(z)=\Phi_{1, \bullet}^{[0, n-1]}(z) .
$$

Proof. Proposition 3.4. in [31] states

$$
\Phi_{n}(z)=\operatorname{det}\left(z-\mathcal{E}_{1, \bullet}^{[0, n-1]}\right) .
$$

The claim follows. 
We also introduce the Aleksandrov polynomials $\Phi_{n}^{\beta}(z)$ by applying the recursion (1.2) to the Verblunsky coefficients $\left\{\beta \alpha_{n}\right\}_{n=0}^{\infty}$. In particular, the polynomial of the second kind is defined by

$$
\Psi_{n}(z)=\Phi_{n}^{-1}(z) .
$$

We have that (Theorem 9.5. in [32])

Lemma 3.7. We have

$$
\Phi_{n}^{\beta}(z)=\Phi_{\beta, \bullet}^{[0, n-1]}(z)
$$

and

$$
\Phi_{n}^{\beta}(z ; \gamma)=\Phi_{\beta, \gamma}^{[0, n-1]}(z)
$$

With these formulas, we obtain the following equality for the absolute value of the Green's function. It would be possible to derive an equality for the Green's function but one would need distinguish between 4 cases depending on if $a$ or $b$ is even or odd.

Proposition 3.8. Let $z \in \mathbb{C}, \beta, \gamma \in \partial \mathbb{D}$, and $a \leq k \leq \ell \leq b$. Then

$$
\left|G_{\beta, \gamma}^{[a, b]}(z ; k, \ell)\right|=\frac{1}{\rho_{k} \rho_{\ell}}\left|\frac{\varphi_{\beta, \bullet}^{[a, k-1]}(z) \varphi_{\bullet, \gamma}^{[\ell+1, b]}(z)}{\varphi_{\beta, \gamma}^{[a, b]}(z)}\right|
$$

Proof. By Cramer's rule and Lemma 3.5, we thus obtain

$$
\left|G_{\beta, \gamma}^{[a, b]}(z ; k, \ell)\right|=\tilde{\rho}_{k+1} \cdots \tilde{\rho}_{\ell-1}\left|\frac{\Phi_{\beta, \bullet}^{[a, k-1]}(z) \Phi_{\bullet, \gamma}^{[\ell+1, b]}(z)}{\Phi_{\beta, \gamma}^{[a, b]}(z)}\right|
$$

The claim now follows from the definition of $\varphi$.

This formula is more awkward than the one for Schrödinger operators, since it involves three different type of polynomials whereas the one for Schrödinger operators only has one (see (2.7) in [5]). Nevertheless it is useful in exactly the same way. We now give the relation of the Green's function to solution of our equation.

Lemma 3.9. Let $\psi$ solve $\mathcal{E} \psi=z \psi$. Then for $a<n<b$

$$
\begin{aligned}
& \psi(n)=G_{\beta, \gamma}^{[a, b]}(z ; n, a) \begin{cases}\left(z \bar{\beta}-\alpha_{a}\right) \psi(a)-\rho_{a} \psi(a+1), & \text { a even } ; \\
\left(z \alpha_{a}-\beta\right) \psi(a)+z \rho_{a} \psi(a+1), & \text { a odd }\end{cases} \\
&+G_{\beta, \gamma}^{[a, b]}(z ; n, b) \begin{cases}\left(z \bar{\gamma}-\alpha_{b}\right) \psi(b)-\rho_{b} \psi(b-1), & b \text { even } ; \\
\left(z \alpha_{b}-\gamma\right) \psi(b)+z \rho_{b-1} \psi(b-1), & b \text { odd }\end{cases}
\end{aligned}
$$

Proof. With $A=\left(z\left(\mathcal{L}_{\beta, \gamma}^{[a, b]}\right)^{*}-\mathcal{M}_{\beta, \gamma}^{[a, b]}\right)$, we have

$$
\varphi(n)=\left\langle A^{-1} \delta_{n}, A \varphi, .\right\rangle
$$

Since, $\left(z(\mathcal{L})^{*}-\mathcal{M}\right) \varphi=0$, we have that for $a+1 \leq n \leq b$ also

$$
A \varphi(n)=0 .
$$

The claim now follows by evaluating this expression for $n \in\{a, b\}$. 
Our next goal will be to introduce transfer matrices and related them to the determinants defined above. We begin with the one-step transfer matrix

$$
A_{z}(\alpha)=\frac{1}{\left(1-|\alpha|^{2}\right)^{\frac{1}{2}}}\left(\begin{array}{cc}
z & -\bar{\alpha} \\
-\alpha z & 1
\end{array}\right) \text {. }
$$

We define the transfer matrix by

$$
T^{[a, b]}(z)=A_{z}\left(\alpha_{b}\right) \cdots A_{z}\left(\alpha_{a}\right) .
$$

Lemma 3.10. We have that

$$
T^{[a, b]}(z)=\frac{1}{2}\left(\begin{array}{cc}
\varphi_{1, \bullet}^{[a, b]}(z)+\varphi_{-1, \bullet}^{[a, b]}(z) & \varphi_{1, \bullet}^{[a, b]}(z)-\varphi_{-1, \bullet \bullet}^{[a, b]}(z) \\
\left(\varphi_{1, \bullet}^{[a, b]}\right)^{*}(z)-\left(\varphi_{-1, \bullet}^{[a, b]}\right)^{*}(z) & \left(\varphi_{1, \bullet}^{[a, b]}\right)^{*}(z)+\left(\varphi_{-1, \bullet}^{[a, b]}\right) *(z)
\end{array}\right)
$$

where $\left(\varphi_{\beta, \gamma}^{[a, b]}\right)^{*}(z)=z^{b-a+1} \overline{\varphi_{\beta, \gamma}^{[a, b]}\left(\bar{z}^{-1}\right)}$.

Proof. The $T_{n}(z)$ in 32 is $T^{[0, n-1]}(z)$ in our notation. We have that

$$
T_{n}(z)=\frac{1}{2}\left(\begin{array}{ll}
\varphi_{n}(z)+\psi_{n}(z) & \varphi_{n}(z)-\psi_{n}(z) \\
\varphi_{n}^{*}(z)-\psi_{n}^{*}(z) & \varphi_{n}^{*}(z)+\psi_{n}^{*}(z)
\end{array}\right)
$$

It follows that

$$
T^{[0, n-1]}(z)=\frac{1}{2}\left(\begin{array}{cc}
\varphi_{1, \bullet}^{[0, n-1]}(z)+\varphi_{-1, \bullet}^{[0, n-1]}(z) & \varphi_{1, \bullet-1]}^{[0, n-\bullet}(z)-\varphi_{-1, \bullet}^{[0, n-1]}(z) \\
\left(\varphi_{1, \bullet}^{[0, n-1]}\right)^{*}(z)-\left(\varphi_{-1, \bullet}^{[0, n-1]}\right)^{*}(z) & \left(\varphi_{1, \bullet}^{[0, n-1]}\right)^{*}(z)+\left(\varphi_{-1, \bullet}^{[0, n-1]}\right)^{*}(z)
\end{array}\right) .
$$

The claim follows using translation invariance.

We thus obtain that

Corollary 3.11. We have that

$$
\left(\begin{array}{c}
\varphi_{\beta, \bullet}^{[a, b]}(z) \\
\left(\varphi_{\beta, \bullet}^{[a, b]}\right)^{*}(z)
\end{array}\right)=T^{[a, b]}(z)\left(\frac{1}{\beta}\right)
$$

and

$$
\varphi_{\beta, \gamma}^{[a, b]}(z)=\frac{1}{\rho_{b}}\left\langle\left(\begin{array}{c}
z \\
-\bar{\gamma}
\end{array}\right), T^{[a, b-1]}(z)\left(\frac{1}{\beta}\right)\right\rangle .
$$

Proof. The first equation is $(3.2 .26)$ in [29]. For the second equation, we have that

$$
\Phi_{\beta, \gamma}^{[a, b]}(z)=z \Phi_{\beta, \bullet}^{[a, b-1]}(z)-\bar{\gamma}\left(\Phi_{\beta, \bullet}^{[a, b-1]}\right)^{*}(z) .
$$

We thus have that

$$
\varphi_{\beta, \gamma}^{[a, b]}(z)=\frac{1}{\rho_{b}}\left(z \varphi_{\beta, \bullet}^{[a, b-1]}(z)-\bar{\gamma}\left(\varphi_{\beta, \bullet}^{[a, b-1]}\right)^{*}(z)\right),
$$

which implies the second equation by the first one.

There is one final object, we need to identify $\varphi_{\bullet, \gamma}^{[a, b]}(z)$. We employ the same strategy as we used in Lemma 3.3 to identify $\mathcal{E}_{\bullet, \gamma}^{(-\infty, 0]}$. Let

$$
\tilde{\alpha}_{n}= \begin{cases}\gamma, & n=-1 ; \\ -\bar{\alpha}_{b-n}, & n \geq 0\end{cases}
$$

Then we have that

$$
\varphi_{\bullet, \gamma}^{[a, b]}(z)=\tilde{\varphi}_{\gamma, \bullet}^{[0, b-a-1]}(z)
$$


Lemma 3.12. We have htat

$$
\left(\begin{array}{c}
\varphi_{\bullet, \gamma}^{[a, b]}(z) \\
\left(\varphi_{\bullet}^{[a, b]}\right)^{*}(z)
\end{array}\right)=\left(\begin{array}{cc}
-\frac{1}{z} & 0 \\
0 & 1
\end{array}\right)\left(T^{[a, b]}(z)\right)^{t}\left(\begin{array}{c}
-z \\
\bar{\gamma}
\end{array}\right) .
$$

Proof. We have that

$$
\left(\begin{array}{cc}
-\frac{1}{z} & 0 \\
0 & 1
\end{array}\right) A_{z}(-\bar{\alpha})^{t}\left(\begin{array}{cc}
-z & 0 \\
0 & 1
\end{array}\right)=A_{z}(\alpha) .
$$

From this the claim follows.

\section{Strictly ergodic CMV matrices}

In this section, we will consider families of CMV operators. This has the advantage that certain formulas will simplify, when viewed probabilistically. Also strict ergodicity simplifies certain statements not available in the ergodic case, in particular [14].

Let $\Omega$ be a compact metric space, $T: \Omega \rightarrow \Omega$ a uniquely ergodic and minimal homeomorphism, and $\mu$ the unique $T$-invariant probability measure. We call $(\Omega, \mu, T)$ strictly ergodic in this case. For a continuous function $f: \Omega \rightarrow \mathbb{D}$, we define the family of Verblunsky coefficients

$$
\alpha_{\omega, n}=f\left(T^{n} \omega\right)
$$

We denote by $\mathcal{E}_{\omega}, \ldots$ the associated objects.

The main example to keep in mind is the $k$-dimensional skew-shift with $\Omega=$ $\mathbb{T}^{k}=(\mathbb{R} / \mathbb{Z})^{k}$

$$
(T x)_{\ell}= \begin{cases}x_{1}+\omega, & \ell=1 \\ x_{\ell}+x_{\ell-1}, & 2 \leq \ell \leq k .\end{cases}
$$

One can then show by induction that

$$
\left(T^{n} x\right)_{\ell}=\left(\begin{array}{c}
n \\
\ell
\end{array}\right) \omega+\left(\begin{array}{c}
n \\
\ell-1
\end{array}\right) x_{1}+\cdots+\left(\begin{array}{l}
n \\
0
\end{array}\right) x_{\ell} .
$$

This map is strictly ergodic, see Proposition 4.7.4. in 11. Then one can realize the Verblunsky coefficient from the introduction as $\alpha_{x, n}$ for $f(x)=\lambda \mathrm{e}^{2 \pi \mathrm{i} x_{k}}$ and a particular choice of $x$.

We now return to our study of the general case of uniquely ergodic and minimal CMV matrices.

Lemma 4.1. We have that $\mathcal{E}_{T x}=\left(S^{*} \mathcal{E}_{x} S\right)^{t}$, where $S$ is the usual forward shift on $\ell^{2}(\mathbb{Z})$. In particular for any $x, y \in \Omega$

$$
\sigma\left(\mathcal{E}_{x}\right)=\sigma\left(\mathcal{E}_{y}\right) .
$$

Proof. The first claim is algebraic. The second claim follows as Lemma 2.2,

For $n \geq 1$, we define the $n$-step (forward) transfer matrix by

$$
T_{x ; n}(z)=A\left(\alpha_{x, n-1}, z\right) \cdots A\left(\alpha_{x, 0}, z\right) .
$$

We note that $T_{x ; n}(z)=T_{x}^{[0, n-1]}(z)$ in the notation of the previous section, and that also $T_{x}^{[a, b]}(z)=T_{T^{a} x ; b-a+1}(z)$. The Lyapunov exponent is defined by

$$
L(z)=\lim _{n \rightarrow \infty} \frac{1}{n} \int_{\mathbb{T}^{k}} \log \left\|T_{n, x}(z)\right\| d x .
$$


We collect its properties

Proposition 4.2. Let $(\Omega, \mu, T)$ be strictly ergodic and $z \in \partial \mathbb{D}$.

(i) $L(z) \geq 0$.

(ii) For almost-every $x \in \mathbb{T}^{k}$, we have as $n \rightarrow \infty$ that

$$
\frac{1}{n} \log \left\|T_{x ; n}(z)\right\| \rightarrow L(z) .
$$

(iii) For every $\varepsilon>0$, there exists $N$ such that for $n \geq N$ and $x \in \mathbb{T}^{K}$ we have

$$
\frac{1}{n} \log \left\|T_{x ; n}(z)\right\| \leq L(z)+\varepsilon .
$$

Proof. (i) follows from $\operatorname{det}(A(\alpha, z))=z$. (ii) is the subadditive ergodic theorem (see Corollary 10.5.25 in [30]). (iii) is Furman's strengthening for uniquely ergodic transformations [14].

The right extension of (4.5) for negative numbers is

$$
T_{x ;-n}(z)=\left(\begin{array}{cc}
-\frac{1}{z} & 0 \\
0 & 1
\end{array}\right) A\left(-\overline{\alpha_{x,-1}}, z\right) \cdots A\left(-\overline{\alpha_{x,-n}}, z\right)\left(\begin{array}{cc}
-z & 0 \\
0 & 1
\end{array}\right)
$$

(where $n \geq 0$ ). This can be seen from (3.27). In particular, one has

$$
L(z)=\lim _{n \rightarrow \infty} \frac{1}{n} \int_{\mathbb{T}^{k}} \log \left\|T_{x ;-n}(z)\right\| d x .
$$

Lemma 4.3. Let $(\Omega, \mu, T)$ be strictly ergodic and $\varepsilon>0$. There exists $C>1$ such that for $n \geq 1$ and $\beta, \gamma \in \partial \mathbb{D}$, we have for $0 \leq k \leq \ell \leq n-1$ that

$$
\left|G_{x ; \beta, \gamma}^{[0, n-1]}(z ; k, \ell)\right| \leq C \frac{\mathrm{e}^{(L(z)+\varepsilon)(k+n-1-\ell)}}{\left|\varphi_{x ; \beta, \gamma}^{[0, n-1]}(z)\right|} .
$$

Proof. By (iii) of Proposition 4.2, there exists $c \geq 1$ such that for any $x \in \Omega$ and $n \geq 1$, we have

$$
\left\|T_{x ; n}(z)\right\| \leq c \mathrm{e}^{(L(z)+\varepsilon) n} .
$$

By (3.23) and (3.27), we obtain that the numerator in Proposition 3.8 is bounded by

$$
c_{2} \cdot \mathrm{e}^{(L(z)+\varepsilon)(n-1-\ell+k)} .
$$

The claim follows.

In particular, we obtain the important theorem

Theorem 4.4. Let $(\Omega, \mu, T)$ be strictly ergodic, $m \in(0, L(E)), \delta>0$, and $\beta_{0}, \gamma_{0} \in$ $\partial \mathbb{D}$. Then for $n$ large enough, there exists $\Omega_{n}$ satisfying $\mu\left(\Omega_{n}\right) \geq 1-\delta$ and for $x \in \Omega_{n}$ there exists

$$
\beta \in\left\{-\beta_{0}, \beta_{0}\right\}, \quad \gamma \in\left\{-\gamma_{0}, \gamma_{0}\right\}
$$

such that for $\frac{n}{3} \leq \ell \leq \frac{2 n}{3}$ and $k \in\{0, n-1\}$

$$
\left|G_{x ; \beta, \gamma}^{[0, n-1]}(z ; k, \ell)\right| \leq \mathrm{e}^{-m|k-\ell|} .
$$


Proof. By (3.24), we have that

$$
\left(\begin{array}{cc}
\varphi_{x ; \beta, \gamma}^{[0, n-1]}(z) & \varphi_{x ;-\beta, \gamma}^{[0, n-1]}(z) \\
\varphi_{x ; \beta,-\gamma}^{[0, n-1]}(z) & \varphi_{x ;-\beta,-\gamma}^{[0, n-1]}(z)
\end{array}\right)=\left(\begin{array}{cc}
z & -\bar{\gamma} \\
z & \bar{\gamma}
\end{array}\right) T_{x, n}(z)\left(\begin{array}{cc}
1 & 1 \\
\beta & -\beta
\end{array}\right)
$$

Since for almost every $x \frac{1}{n} \log \left\|T_{x, n}(z)\right\| \geq L(z)(1-\varepsilon)$ for $n$ large enough, the claim follows.

\section{Rotationally inVARiance AND the Proof of TheOREM 1.2}

We begin this section by investigating what happens if one rotates the Verblunsky coefficients, which is essentially what we used to prove Theorem 1.1. We have the following important proposition

Proposition 5.1. Let $\beta, \gamma \in \overline{\mathbb{D}}, a<b$ integers, and $x, y \in \mathbb{T}$ and define

$$
\tilde{\alpha}_{n}=e(n x+y) \alpha_{n}, \quad \tilde{\beta}=e((a-1) x+y) \beta, \quad \tilde{\gamma}=e(b x+y) \gamma .
$$

Then $\mathcal{E}_{\beta, \gamma}^{[a, b]}$ and $e(x) \widetilde{\mathcal{E}}_{\tilde{\beta}, \tilde{\gamma}}^{[a, b]}$ are unitarily equivalent.

Here and in the following, we abbreviate $e(x)=\mathrm{e}^{2 \pi \mathrm{i} x}$. We will prove this proposition in the case of $a$ and $b$ finite. It is interesting if it holds for $a, b$ possibly infinite. An inspection of the proof of Proposition 5.1 shows that it also holds for whole line CMV operators with pure point spectrum. In particular, it implies that in the case $k=2$, all the operators $\mathcal{E}_{x}$ defined by the skew-shift are unitarily equivalent. Since the Jitomirskaya-Simon [19] argument applies in our case, all the $\mathcal{E}_{x}$ have purely singular continuous spectrum. For the proof of this proposition, we need the following lemma

Lemma 5.2. Pick some $u_{a} \in \partial \mathbb{D}$ and define a sequence recursively by

$$
u_{n}= \begin{cases}u_{n-1} e(-(n-1) x-y), & n \text { even } \\ u_{n-1} e((n-1) x+y), & n \text { odd } .\end{cases}
$$

Furthermore, we define the multiplication operators

$$
U \psi(n)=u_{n} \psi(n), \quad V \psi(n)=\left\{\begin{array}{ll}
u_{n-1} \psi(n), & n \text { even } \\
u_{n-1} e(-x) \psi(n), & n \text { odd }
\end{array} .\right.
$$

Then for $\tilde{z}=e(-x) z$

$$
\left(\tilde{z}\left(\widetilde{\mathcal{L}}_{\tilde{\beta}, \tilde{\gamma}}^{[a, b]}\right)^{*}-\widetilde{\mathcal{M}}_{\tilde{\beta}, \tilde{\gamma}}^{[a, b]}\right) U=V\left(z\left(\mathcal{L}_{\beta, \gamma}^{[a, b]}\right)^{*}-\mathcal{M}_{\beta, \gamma}^{[a, b]}\right) .
$$

Proof. A computation shows for $n$ even that

$$
\tilde{z} \tilde{\alpha}_{n}+\tilde{\alpha}_{n-1}=e((n-1) x+y)\left(z \alpha_{n}+\alpha_{n-1}\right)
$$

and for $n$ odd

$$
\tilde{z} \overline{\tilde{\alpha}_{n-1}}+\overline{\tilde{\alpha}_{n}}=e(-n x-y)\left(z \overline{\alpha_{n-1}}+\overline{\alpha_{n}}\right) .
$$

By Lemma 3.5, we thus obtain that for $n$ even we have that

$$
\begin{aligned}
\left(\tilde{z}\left(\widetilde{\mathcal{L}}_{\beta, \gamma}^{[a, b]}\right)^{*}-\widetilde{\mathcal{M}}_{\beta, \gamma}^{[a, b]}\right) U \psi(n) & =e(-x) z \rho_{n} u_{n+1} \psi(n+1)-\rho_{n-1} u_{n-1} \psi(n-1) \\
& +u_{n} e((n-1) x+y)\left(z \alpha_{n}+\alpha_{n-1}\right) \psi(n) .
\end{aligned}
$$


Since $u_{n}=e(-(n-1) x-y) u_{n-1}$ and $u_{n+1}=e(x) u_{n-1}$, the claimed equality follows for $n$ even. Similarly for $n$ odd

$$
\begin{aligned}
\left(\tilde{z}\left(\widetilde{\mathcal{L}}_{\beta, \gamma}^{[a, b]}\right)^{*}-\widetilde{\mathcal{M}}_{\beta, \gamma}^{[a, b]}\right) U \psi(n) & =-\rho_{n} u_{n+1} \psi_{n+1}+e(-x) z \rho_{n-1} u_{n-1} \psi_{n-1} \\
& -e(-n x-y)\left(z \overline{\alpha_{n-1}}+\overline{\alpha_{n}}\right) u_{n} \psi(n) .
\end{aligned}
$$

Since $u_{n+1}=u_{n} \cdot e(-n x-y)$ and $u_{n}=e((n-1) x+y) u_{n-1}$, we obtain the claim.

Proof of Proposition 5.1. Since the spectra of $\mathcal{E}_{\beta, \gamma}^{[a, b]}$ and $e(x) \widetilde{\mathcal{E}}_{\tilde{\beta}, \tilde{\gamma}}^{[a, b]}$ are simple, it suffices to show that they are the same. If $\mathcal{E}_{\beta, \gamma}^{[a, b]} \psi=z \psi$ for $\psi \neq 0$, we have that $\left(z\left(\mathcal{L}_{\beta, \gamma}^{[a, b]}\right)^{*}-\mathcal{M}_{\beta, \gamma}^{[a, b]}\right) \psi=0$. Hence, by the previous lemma also that

$$
\left(\tilde{z}\left(\widetilde{\mathcal{L}}_{\tilde{\beta}, \tilde{\gamma}}^{[a, b]}\right)^{*}-\widetilde{\mathcal{M}}_{\tilde{\beta}, \tilde{\gamma}}^{[a, b]}\right) \varphi=0
$$

for $\varphi=U \psi \neq 0$. Hence, we also have that

$$
\left(z-e(x) \widetilde{\mathcal{E}}_{\tilde{\beta}, \tilde{\gamma}}^{[a, b]}\right) \varphi=0
$$

which implies the claim.

We will now begin drawing conclusions from Proposition 5.1. For the sake of concreteness, we will only consider the Verblunsky coefficients given by

$$
\alpha_{x, n}=\lambda \mathrm{e}^{2 \pi \mathrm{i}\left(T^{n} x\right)_{k}}
$$

where $x \in \mathbb{T}^{k}, \lambda \in \mathbb{D} \backslash\{0\}$, and $T: \mathbb{T}^{k} \rightarrow \mathbb{T}^{k}$ is the $k$ dimensional skew-shift defined in (4.2).

For $\theta_{1}, \theta_{2} \in \mathbb{T}$, we denote by $P_{\left[\theta_{1}, \theta_{2}\right]}$ the spectral projection on the $\operatorname{arc}\left\{\mathrm{e}^{2 \pi \mathrm{it}}\right.$ : $\left.t \in\left[\theta_{1}, \theta_{2}\right](\bmod 1)\right\}$. We then have that

Theorem 5.3. Let $\beta_{0}, \gamma_{0} \in \partial \mathbb{D}$ and define

$$
\beta_{x}=\beta_{0} \frac{\alpha_{x,-1}}{\left|\alpha_{x,-1}\right|}, \quad \gamma_{x}=\gamma_{0} \frac{\alpha_{x, n-1}}{\left|\alpha_{x, n-1}\right|} .
$$

Then

$$
\frac{1}{n} \int_{\mathbb{T}^{k}} \operatorname{tr}\left(P_{\left[\vartheta_{1}, \vartheta_{2}\right]} \mathcal{E}_{x ; \beta_{x}, \gamma_{x}}^{[0, n-1]}\right) d x=\left|\theta_{2}-\theta_{1}\right| .
$$

Proof. We will show this is true, when only performing the $x_{k-1}$ integral. Let $s=x_{k-1}$. Then changing $s$ amounts to changing $x$ in Proposition 5.1. Hence, the eigenvalues are given by

$$
\mathrm{e}^{2 \pi \mathrm{i}\left(\theta_{1}-s\right)}, \ldots, \mathrm{e}^{2 \pi \mathrm{i}\left(\theta_{N}-s\right)}
$$

as $s$ varies. This implies the claim.

It is easy to infer from this that the integrated density of states is just given by the normalized Lebesgue measure. We now come to

Theorem 5.4. For $z \in \partial \mathbb{D}$, we have that

$$
\gamma(z)=-\frac{1}{2} \log \left(1-|\lambda|^{2}\right) .
$$

Proof. This can be shown as in Theorem 12.6.2. in [30]. 
Proof of Theorem 1.2. For $\theta \in \mathbb{T}$, we have

$$
\alpha_{\tilde{x}, n}=\mathrm{e}^{2 \pi \mathrm{i} \theta} \alpha_{x, n}
$$

where

$$
\tilde{x}_{\ell}=\left\{\begin{array}{ll}
x_{\ell}, & 1 \leq \ell \leq k-1 ; \\
x_{k}+\theta, & \ell=k .
\end{array} .\right.
$$

The claim now follows from Theorem 12.6.1. in [30].

Proof of Proposition 2.3. If $z \in \sigma_{\text {ess }}(\mathcal{C})$ then there exists a sequence $\psi_{j} \in \ell^{2}(\mathbb{N})$ such that $\left\|\psi_{j}\right\|=1, \psi_{j} \rightarrow 0$ weakly, and $\left\|(\mathcal{C}-z) \psi_{j}\right\| \rightarrow 0$. In particular, we have for any $N \geq 1$ fixed

$$
\sum_{n=1}^{N}\left|\psi_{j}(n)\right|^{2} \rightarrow 0
$$

By Lemma 5.2 with $x=2 \pi \eta, y=0$, we obtain that $\varphi_{j}=U \psi_{j}$ satisfy $\varphi_{j} \rightarrow 0$ weakly and

$$
\left\|\left(\widetilde{\mathcal{C}}-\mathrm{e}^{-2 \pi \mathrm{i} \eta} z\right) \varphi_{j}\right\| \rightarrow 0 .
$$

Hence, the claim follows.

\section{Eigenvalue statistics and the proof of Theorem 1.3}

Since we will focus on the case $k=2$, it will be convenient to introduce the skew-shift $T: \mathbb{T}^{2} \rightarrow \mathbb{T}^{2}$ by

$$
T(x, y)=(x+2 \omega, x+y) \quad(\bmod 1) .
$$

One easily checks that this is equivalent to (4.2) and that

$$
T^{n}(x, y)=(x+2 n \omega, y+n x+n(n-1) \omega) \quad(\bmod 1) .
$$

Then our Verblunsky coefficients are given by

$$
\alpha_{x, y ; n}=\lambda e(y+n x+n(n-1) \omega),
$$

where we use the abbreviation $e(t)=\mathrm{e}^{2 \pi \mathrm{i} t}$.

The main goal of this section is to prove the following theorem, which will imply Theorem 1.3 .

Theorem 6.1. Assume $\omega$ satisfies (1.8). Let $x, y \in \mathbb{T}$ and $\beta, \gamma \in \partial \mathbb{D}$. There exists $\sigma>0$ such that for $N$ sufficiently large, there exist $\theta_{1}^{N}, \ldots, \theta_{N}^{N}$ and $\vartheta^{N}$ such that

$$
\sigma\left(\mathcal{E}_{x, y ; \beta, \gamma}^{[0, N-1]}\right)=\left\{\mathrm{e}^{2 \pi \mathrm{i} \theta_{1}^{N}}, \ldots, \mathrm{e}^{2 \pi \mathrm{i} \theta_{N}^{N}}\right\}
$$

and

$$
\frac{1}{N} \#\left\{n: \quad\left\|\theta_{n}^{N}-\vartheta^{N}+2 n \omega\right\|>\frac{1}{N^{1+\sigma}}\right\} \leq \frac{1}{N^{\sigma}} .
$$

In order to see how this implies Theorem 1.3, we need to introduce some more notation related to the Laplace functional. Given $N$ points $x_{1}^{N}, \ldots, x_{N}^{N} \in \mathbb{T}$, we define for $\theta \in \mathbb{T}$

$$
\left[-\frac{1}{2}, \frac{1}{2}\right) \ni x_{n}^{N}(\theta)=x_{n}^{N}-\theta \quad(\bmod 1) .
$$


Then their Laplace functional is defined by

$$
\mathfrak{L}_{x^{N}, N}(f)=\int_{\mathbb{T}} \exp \left(-\sum_{n=1}^{N} f\left(N x_{n}^{N}(\theta)\right)\right) d \theta
$$

where $f$ is a continuous, compactly supported, and positive function. If $\underline{x}=$ $\left\{\left\{x_{n}^{N}\right\}_{n=1}^{N}\right\}_{N=1}^{\infty}$ is a sequence of vectors, we denote

$$
\mathfrak{L}_{\underline{x}, N}(f)=\mathfrak{L}_{x^{N}, N}(f) .
$$

Theorem 1.3 follows by applying (iv) of the next lemma to the sequences

$$
\underline{\theta}=\left\{\left\{\theta_{n}^{N}\right\}_{n=1}^{N}\right\}_{N=1}^{\infty}, \quad \underline{\vartheta}=\left\{\left\{\vartheta^{N}-2 n \omega\right\}_{n=1}^{N}\right\}_{N=1}^{\infty}
$$

Lemma 6.2. Let $f: \mathbb{R} \rightarrow \mathbb{R}$ be a positive, continuous, and compactly supported function, $\underline{x}=\left\{\left\{x_{n}^{N}\right\}_{n=1}^{N}\right\}_{N=1}^{\infty}$ and $\underline{y}$ be sequences of vectors in $\mathbb{T}$.

(i) Let $c>0$ and $A>1$, then

$$
\left|\left\{\theta \in \mathbb{T}: \quad \#\left\{1 \leq n \leq N: \quad N x_{n}^{N}(\theta) \in[-c, c]\right\} \geq A\right\}\right| \leq \frac{2 c}{N} .
$$

(ii) If $\max _{1 \leq n \leq N} N\left\|x_{n}^{N}-y_{n}^{N}\right\| \rightarrow 0$ then

$$
\left|\mathfrak{L}_{\underline{x}, N}(f)-\mathfrak{L}_{\underline{y}, N}(f)\right| \rightarrow 0 .
$$

(iii) If

$$
\frac{1}{N} \#\left\{1 \leq n \leq N: \quad x_{n}^{N} \neq y_{n}^{N}\right\} \rightarrow 0
$$

then

$$
\left|\mathfrak{L}_{\underline{x}, N}(f)-\mathfrak{L}_{\underline{y}, N}(f)\right| \rightarrow 0 .
$$

(iv) If for every $\varepsilon>0$

$$
\frac{1}{N} \#\left\{1 \leq n \leq N: \quad\left\|x_{n}^{N}-y_{n}^{N}\right\| \geq \frac{\varepsilon}{N}\right\} \rightarrow 0
$$

then

$$
\left|\mathfrak{L}_{\underline{x}, N}(f)-\mathfrak{L}_{\underline{y}, N}(f)\right| \rightarrow 0 .
$$

Proof of (i). Follows from

$$
\int_{-\frac{1}{2}}^{\frac{1}{2}} \sum_{n=1}^{N} \chi_{\left[-\frac{c}{N}, \frac{c}{N}\right]}\left(x_{n}^{N}(\theta)\right) d \theta=2 c .
$$

and Markov's inequality.

Proof of (ii). Let $\varepsilon>0$. Since $f$ is compactly supported, we have $\operatorname{supp}(f) \subseteq[-c, c]$. Let $A=\left\lceil\frac{2 c}{10 \varepsilon}\right\rceil$. By (i), there exists a set $I \subseteq \mathbb{T}$ such that for $\theta \in I$

$$
\#\left\{1 \leq n \leq N: \quad N x_{n}(\theta) \in[-c, c] \text { or } N y_{n}(\theta) \in[-c, c]\right\} \leq A
$$

and $|\mathbb{T} \backslash I| \leq \frac{\varepsilon}{2}$. By assumption, $f$ is uniformly continuous, so there exists a $\delta>0$ such that $|f(x)-f(y)| \leq \frac{\varepsilon}{2 A}$ for $|x-y|<\delta$. Choose $N$ so large that

$$
\max _{1 \leq n \leq N} N\left\|x_{n}^{N}-y_{n}^{N}\right\|<\delta .
$$


Then we clearly have that $\left|N x_{n}(\theta)-N y_{n}(\theta)\right|<\delta$, and thus that for $\theta \in I$.

$$
\left|\sum_{n=0}^{N-1} f\left(N x_{n}(\theta)\right)-\sum_{n=0}^{N-1} f\left(N y_{n}(\theta)\right)\right|<\frac{\varepsilon}{2} .
$$

The claim follows.

Proof of (iii). (ii) follows from the set of $\theta$ for which

$$
\sum_{n=1}^{N} f\left(N y_{n}^{N}(\theta)\right) \neq \sum_{n=1}^{N} f\left(N x_{n}^{N}(\theta)\right)
$$

having vanishing measure as $N \rightarrow \infty$.

Proof of (iv). By assumption, there exists $\varepsilon_{N} \rightarrow 0$ such that

Define

$$
\frac{1}{N} \#\left\{1 \leq n \leq N: \quad\left\|x_{n}^{N}-y_{n}^{N}\right\| \geq \frac{\varepsilon_{N}}{N}\right\} \rightarrow 0 .
$$

$$
\tilde{x}_{n}^{N}= \begin{cases}y_{n}^{N}, & \left\|x_{n}^{N}-y_{n}^{N}\right\| \geq \frac{\varepsilon_{N}}{N} \\ x_{n}^{N}, & \text { otherwise. }\end{cases}
$$

Then $x^{N}$ and $\tilde{x}^{N}$ satisfy the assumptions of (i) and $\tilde{x}^{N}$ and $y^{N}$ the ones of (ii). The claim follows.

We now begin the proof of Theorem 6.1 (1.8) implies that there exists some $c>0$ such that

$$
\|q \omega\| \geq \frac{c}{q^{\tau}}
$$

for all positive integers $q$. The following theorem will be essential to our proof and proven only in the next section.

Theorem 6.3. There is a constant $\sigma \in(0,1)$. Let $\eta \geq 1, N$ sufficiently large, $\beta, \gamma \in \partial \mathbb{D}$ and $x, y \in \mathbb{T}$. There exists a normalized $\psi \in \ell^{2}(\{0, \ldots, N-1\})$ such that $\psi(n)=0$ for $n \geq N^{\sigma}, n=0,1$ and $z=\mathrm{e}^{2 \pi \mathrm{i} \vartheta}$ such that

$$
\left\|\left(\mathcal{E}_{x, y ; \beta, \gamma}^{[0, N-1]}-z\right) \psi\right\| \leq\left(\frac{1}{N}\right)^{\eta}
$$

Define $\vartheta_{k}=\vartheta-2 \omega k$ and $z_{k}=e\left(\vartheta_{k}\right)$.

Lemma 6.4. Let $\varepsilon>0$. If (6.16) holds, then for $N$ large enough and $k \neq \tilde{k} \in$ $\{0, \ldots, N-1\}$ we have

$$
\left|z_{k}-z_{\tilde{k}}\right| \geq \frac{1}{N^{\tau+\varepsilon}}
$$

Proof. Clear.

Proof of Theorem 6.1. We let $\eta=\tau+2 \varepsilon$ in Theorem 6.3. With $u_{n}$ the appropriate factors as given in Lemma 5.2 , we define the test functions

$$
\psi_{k}(n)= \begin{cases}u_{n} \psi(n-k), & k \leq n \leq k+N^{\sigma} \\ 0, & \text { otherwise. }\end{cases}
$$

We then have for $0 \leq k \leq N-N^{\sigma}$ that

$$
\left\|\left(\mathcal{E}_{x, y ; \beta, \gamma}^{[0, N-1]}-z_{k}\right) \psi_{k}\right\| \leq \frac{1}{N^{\eta}}
$$


Hence, there is some eigenvalue $\mathrm{e}^{2 \pi \mathrm{i} \theta_{\ell_{k}}}$ such that

$$
\left\|\theta_{\ell_{k}}-\vartheta_{k}\right\| \leq \frac{1}{N^{\eta}}
$$

By the previous lemma, we must have $\ell_{k} \neq \ell_{\tilde{k}}$ for $k \neq \tilde{k}$. The claim then follows upon reordering the $\theta_{\ell}$.

\section{Proof of Theorem 6.3}

Let $L=\left\lfloor\frac{1}{3} N^{\sigma}\right\rfloor$. If we show that for every $(x, y) \in \mathbb{T}^{2}$, there exists a normalized vector $\psi$ and $z \in \partial \mathbb{D}$ such that

$$
\left\|\left(\mathcal{E}_{x, y ; \beta, \gamma}^{[-L, L]}-z\right) \psi\right\| \leq \frac{1}{N^{C}}
$$

then Theorem 6.3 follows. We will show this modified claim, since it is notationally somewhat simpler to deal with.

Since $\mathcal{E}_{x, y ; \beta, \gamma}^{[-L, L]}$ has $2 L+1$ eigenvalues, there exists $z \in \mathbb{D}$ and $\|\psi\|=1$ such that

$$
\mathcal{E}_{x, y ; \beta, \gamma}^{[-L, L]} \psi=z \psi, \quad|\psi(0)|^{2} \geq \frac{1}{2 L+1}
$$

We will prove in the following section

Theorem 7.1. There exists $\eta>0$ such that for every $C \geq 1$, we have for $L$ large enough and $M=\left\lfloor L^{\eta}\right\rfloor$ that there exist

$$
-\frac{2}{3} L \leq k_{-} \leq-\frac{1}{3} L, \quad \frac{1}{3} L \leq k_{+} \leq \frac{2}{3} L
$$

such that for

$$
k \in\left\{k_{-}-C M, \ldots, k_{-}+C M\right\} \cup\left\{k_{+}-C M, \ldots, k_{+}+C M\right\}
$$

we have that there exist $\beta, \gamma \in \partial \mathbb{D}$ such that for $|k-\ell| \leq \frac{M}{2}$ we have

$$
\left|G_{x, y ; \beta, \gamma}^{[k-M, k+M]}(z ; \ell, k-M)\right|,\left|G_{x, y ; \beta, \gamma}^{[k-M, k+M]}(z ; \ell, k+M)\right| \leq \frac{1}{M} .
$$

Define

$$
\mathcal{K}_{t}=\left\{k_{-}-t M, \ldots, k_{-}+t M\right\} \cup\left\{k_{+}-t M, \ldots, k_{+}+t M\right\} .
$$

Using Lemma 3.9 combined with the estimate from the previous theorem, we can conclude for $k \in K_{C}$ and $|\ell-k| \leq \frac{M}{2}$ that

$$
|\psi(\ell)| \leq \frac{4}{M}
$$

where we used the trivial estimate $|\psi(n)| \leq 1$ $\frac{M}{2}$

We can iterate this to obtain for $s=1, \ldots, C$ that for $k \in K_{C-s+1}$ and $|\ell-k| \leq$

$$
|\psi(\ell)| \leq\left(\frac{4}{M}\right)^{s}
$$

In particular, we obtain that

$$
\left|\psi\left(k_{-}\right)\right|,\left|\psi\left(k_{+}\right)\right| \leq\left(\frac{4}{M}\right)^{C} .
$$


Define a test function $\varphi$ by

$$
\varphi(n)= \begin{cases}\psi(n), & k_{-} \leq n \leq k_{+} \\ 0, & \text { otherwise }\end{cases}
$$

We have that

$$
\left\|\left(\mathcal{E}_{x, y ; \beta, \gamma}^{[-L, L]}-z\right) \varphi\right\| \leq\left(\frac{8}{L}\right)^{C \eta}
$$

and thus Theorem 6.3 follows.

\section{Decay of the Green's function: Proof of Theorem 7.1}

Theorem 4.4 states that $m=L(z)>0$ implies that for $N$ large enough there exists a set $B_{N} \subseteq \mathbb{T}^{2}$ with

(i) $\left|B_{N}\right| \rightarrow 0$.

(ii) For $(x, y) \in \mathbb{T}^{2} \backslash B_{N}$, there exists

$$
\beta \in\left\{-\frac{\alpha_{-N-1}}{\left|\alpha_{-N-1}\right|}, \frac{\alpha_{-N-1}}{\left|\alpha_{-N-1}\right|}\right\}, \quad \gamma \in\left\{\frac{\alpha_{N}}{\left|\alpha_{N}\right|},-\frac{\alpha_{N}}{\left|\alpha_{N}\right|}\right\}
$$

such that for $|k| \leq \frac{N}{2}$, we have

$$
\left|G_{x, y ; \beta, \gamma}^{[-N, N]}(z ;-N, k)\right| \leq \mathrm{e}^{-\frac{m}{4}|k+N|}, \quad\left|G_{x, y ; \beta, \gamma}^{[-N, N]}(z ; N, k)\right| \leq \mathrm{e}^{-\frac{m}{4}|N-k|} .
$$

We will first need the following lemma.

Lemma 8.1. Let $\sigma>0$, there exists a constant $C>1$ such that for $N \geq 1$, there exists a set $B_{N}^{2}$ such that

$$
\left|B_{N}^{2}\right| \leq \frac{C}{N^{\sigma}}
$$

and for

$$
\beta \in\left\{-\frac{\alpha_{-N-1}}{\left|\alpha_{-N-1}\right|}, \frac{\alpha_{-N-1}}{\left|\alpha_{-N-1}\right|}\right\}, \quad \gamma \in\left\{\frac{\alpha_{N}}{\left|\alpha_{N}\right|},-\frac{\alpha_{N}}{\left|\alpha_{N}\right|}\right\}
$$

and $x, y \in \mathbb{T}^{2} \backslash B_{N}^{2}$ we have

$$
\left\|\left(z\left(\mathcal{L}_{x, y ; \beta, \gamma}^{[-N, N]}\right)^{*}-\mathcal{M}_{x, y ; \beta, \gamma}^{[-N, N]}\right)^{-1}\right\| \leq N^{1+\sigma}
$$

Proof. This is a consequence of Theorem 5.3 .

In summary, we have extracted the following statement

Proposition 8.2. Let $\sigma>0$. For $N \geq 1$ large enough, there exists $\Omega_{N} \subseteq \mathbb{T}^{2}$ such that

$$
\lim _{N \rightarrow \infty}\left|\mathbb{T}^{2} \backslash \Omega_{N}\right|=0
$$

For each $(x, y) \in \Omega_{N}$ and

$$
|\tilde{x}-x| \leq \frac{1}{N^{2(1+2 \sigma)}}, \quad|\tilde{y}-y| \leq \frac{1}{N^{1+2 \sigma}},
$$

we have that there exists

$$
\beta \in\left\{-\frac{\alpha_{-N-1}}{\left|\alpha_{-N-1}\right|}, \frac{\alpha_{-N-1}}{\left|\alpha_{-N-1}\right|}\right\}, \quad \gamma \in\left\{\frac{\alpha_{N}}{\left|\alpha_{N}\right|},-\frac{\alpha_{N}}{\left|\alpha_{N}\right|}\right\}
$$


such that for $|k| \leq \frac{N}{2}$, we have

$$
\left|G_{\tilde{x}, \tilde{y} ; \beta, \gamma}^{[-N, N]}(z ;-N, k)\right| \leq \frac{1}{N}, \quad\left|G_{\tilde{x}, \tilde{y} ; \beta, \gamma}^{[-N, N]}(z ; N, k)\right| \leq \frac{1}{N} .
$$

Proof. A computation shows that

$$
\left\|\mathcal{L}_{x, y ; \beta, \gamma}^{[-N, N]}-\mathcal{L}_{\tilde{x}, \tilde{y} ; \beta, \gamma}^{[-N, N]}\right\| \lesssim N|x-\tilde{x}|+|y+\tilde{y}| \leq \frac{1}{N^{\frac{\sigma}{2}}}
$$

for $N$ large enough and a similar result for $\mathcal{M}_{\tilde{x}, \tilde{y} ; \beta, \gamma}^{[-N, N]}$. The result now follows from

$$
B^{-1}-A^{-1}=B^{-1}(A-B) A^{-1}
$$

and some computations.

Let $X_{N}=\left\lceil N^{2(1+2 \sigma)}\right\rceil, Y_{N}=\left\lceil N^{1+2 \sigma}\right\rceil$. We partition $\mathbb{T}^{2}$ into $X_{N} \cdot Y_{N} \lesssim N^{3(1+2 \sigma)}$ boxes of side length $\frac{1}{X_{N}}$ and $\frac{1}{Y_{N}}$. We call a box $I_{\ell}$ bad if

$$
\Omega_{N} \cap I_{\ell}=\emptyset
$$

and good otherwise. We note that if $(x, y)$ is in a good box, then for $|k| \leq \frac{N}{2}$

$$
\left|G_{x, y ; \beta, \gamma}^{[-N, N]}(z ; k, \pm N)\right| \leq \frac{1}{N}
$$

for some $\beta, \gamma \in \partial \mathbb{D}$. We now given an upper bound on the number of iterates of $T^{j}(x, y)$ that land in any bad box. We will show the following theorem in Appendix A]. For $\varepsilon, \delta>0$, denote by $B_{\varepsilon, \delta} \subseteq \mathbb{T}^{2}$ the set

$$
B_{\varepsilon, \delta}=\left\{(x, y) \in \mathbb{T}^{2}: \quad\|x\| \leq \varepsilon,\|y\| \leq \delta\right\} .
$$

Theorem 8.3. Assume (1.8) and let $\delta>0, \varepsilon>0, N \geq 1$. There exists $L_{0}=$ $L_{0}(\sigma, \omega) \geq 1$ such that for any $x, y \in \mathbb{T}$ there exists $0 \leq \ell_{0} \leq N$ such that for $L \geq L_{0} \delta^{-4} \varepsilon^{-9}$

$$
\#\left\{0 \leq \ell \leq \frac{L_{0}}{N}: \quad T^{\ell N}(x, y) \in B_{\varepsilon, \delta}\right\} \leq 10 \varepsilon \delta \frac{L}{N}
$$

We now obtain that for $L \geq N^{15}$ and $N$ large enough, we have for some $0 \leq$ $\ell_{0} \leq N-1$

$$
\#\left\{\left\lfloor\frac{1}{3 N} L+\ell_{0}\right\rfloor \leq \ell \leq \frac{2}{3 N} L: \quad T^{\ell N}(x, y) \text { in fixed bad box }\right\} \leq \frac{10 L / N}{N^{3(1+\sigma)}} .
$$

Since

$$
\#\{\text { bad boxes }\} \leq \delta_{N} N^{3(1+\sigma)}
$$

with $\delta_{N} \rightarrow 0$ as $N \rightarrow \infty$, we obtain for $L \geq N^{15}$ that

$$
\#\left\{\left\lfloor\frac{1}{3 N} L+\ell_{0}\right\rfloor \leq \ell \leq \frac{2}{3 N} L: \quad T^{\ell N}(x, y) \text { in some bad box }\right\} \leq \delta_{N} \frac{L}{N}
$$

for $\delta_{N} \rightarrow 0$ as $N \rightarrow \infty$.

Proof of Theorem 7.1. We just give the argument for $k_{+}$. Choose $\delta_{N} \leq \frac{1}{10 C}$. Now divide $\left[\left\lfloor\frac{1}{3 N} L+\ell_{0}\right\rfloor, \frac{2}{3 N} L\right]$ into segments of length $3 C$. Then at most $\delta_{N} \frac{L}{N}$ of them can contain an iterate that lands in a bad box, but there are $\frac{1}{C} \frac{L}{3 N}=10 \delta_{N} \frac{L}{3 N}$ many of them. Hence, we must have at least one, where our conclusion holds. 


\section{Appendix A. Dynamics of the SKeW-ShifT}

In this section, we will discuss quantitative recurrence results for the skew-shift. The discussing here follows the one in Chapters 10 and 11 in [23].

Theorem A.1. Assume (6.16) and let $\sigma>0$. Then for a constant $C=C(c, \tau, \sigma)>$ 0 we have for $L \geq 1$

$$
\#\left\{1 \leq \ell \leq L: \quad T^{\ell}(x, y) \in B_{\varepsilon, \delta}\right\} \leq 5 \varepsilon \delta L+C\left(\frac{1}{\varepsilon}\right)^{1+\sigma} L^{\frac{1}{2}+\sigma} .
$$

Proof of Theorem 8.3. Let $\sigma=\frac{1}{4}$ in the previous theorem. Then we have that

$$
\#\left\{1 \leq \ell \leq L: \quad T^{\ell}(x, y) \in B_{\varepsilon, \delta}\right\} \leq 10 \varepsilon \delta L
$$

if $C\left(\frac{1}{\varepsilon}\right)^{\frac{5}{4}} L^{\frac{3}{4}} \leq 5 \varepsilon \delta L$ or equivalently

$$
L^{\frac{1}{4}} \geq \frac{C}{5 \delta} \cdot \frac{1}{\varepsilon^{\frac{9}{4}}} .
$$

Next divide $1 \leq \ell \leq L$ into $N$ arithmetic progressions of the form $\left\{\ell_{0}+\ell N\right\}_{\ell=0}^{L / N}$ for $\ell_{0} \in\{1, \ldots, L\}$. Then at least one of them must contain less than $\frac{10 \varepsilon \delta L}{N}$ elements.

We now begin to prove Theorem A.1

Lemma A.2. There exists a trigonometric polynomial $P$ given by

$$
P(x, y)=\sum_{|j| \leq \frac{2}{\varepsilon}|k| \leq \frac{2}{\delta}} P_{j, k} e(j x+k y)
$$

such that $\left|P_{j, k}\right| \leq 5 \varepsilon \delta$ and

$$
\chi_{B_{\varepsilon, \delta}} \leq P,
$$

where $\chi_{A}$ denotes the characteristic function of $A \subseteq \mathbb{T}^{2}$.

Proof. Follows by using Selberg polynomials, see Chapter 2 in [26].

We compute that

$$
\begin{aligned}
\#\{1 \leq \ell \leq L: \quad & \left.T^{\ell}(x, y) \in B_{\varepsilon, \delta}\right\} \leq \sum_{\ell=1}^{L} P\left(T^{\ell}(x, y)\right) \\
& \leq 5 \varepsilon \delta L+5 \varepsilon \delta \sum_{|j| \leq \frac{2}{\varepsilon}|k| \leq \frac{2}{\delta}} \sum_{\ell=1}^{L} e\left(j \cdot 2 \ell \omega+k x \ell-k \omega \ell+k \omega \ell^{2}\right) \mid .
\end{aligned}
$$

To finish the proof of Theorem A.1 we will need the next two bounds.

Lemma A.3. We have

$$
\left|\sum_{\ell=1}^{L} e(\ell \cdot \omega)\right| \leq \frac{1}{2\|\omega\|} .
$$

and for $\sigma>0$, there exists $C=C(\sigma)>0$ such that for any $t \in \mathbb{R}$

$$
\left|\sum_{\ell=1}^{L} e\left(t \ell+\omega \ell^{2}\right)\right| \leq \frac{C L^{\frac{1}{2}+\sigma}}{\|\omega\|} .
$$


Proof. See Chapter 3 in [26.

Proof of Theorem A.1. From the previous lemma and the computation preceeding it, we obtain

$$
\#\left\{1 \leq \ell \leq L: \quad T^{\ell}(x, y) \in B_{\varepsilon, \delta}\right\} \leq 5 \varepsilon \delta L+C L^{\frac{1}{2}+\sigma} \sup _{1 \leq k \leq \frac{2}{\varepsilon}} \frac{1}{\|k \omega\|} .
$$

The claim now follows by (1.8).

\section{ACKNOWLEDGEMENTS}

The key realization that Proposition 5.1 and Lemma 5.2 hold, came to me during discussions with Darren Ong. Furthermore, I am thankful to Maxim Zinchenko for correspondence, which clarified issues related to (3.27).

\section{REFERENCES}

[1] A. Avila, J. Bochi, D. Damanik, Cantor spectrum for Schrödinger operators with potentials arising from generalized skew-shifts. Duke Math. J. 146 (2009), 253-280.

[2] A. Avila, J. Bochi, D. Damanik, Opening Gaps in the Spectrum of Strictly Ergodic Schrödinger Operators. JEMS, (to appear).

[3] A. Avila, S. Jitomirskaya, Solving the Ten Martini Problem. Lecture Notes in Physics 690 (2006), 5-16.

[4] A. Avila, S. Jitomirskaya, The Ten Martini Problem. Ann. of Math. 170 (2009), 303-342.

[5] J. Bourgain, Green's function estimates for lattice Schrödinger operators and applications, Annals of Mathematics Studies, 158. Princeton University Press, Princeton, NJ, 2005. $\mathrm{x}+173 \mathrm{pp}$.

[6] J. Bourgain, Positive Lyapounov exponents for most energies, Geometric aspects of functional analysis, 37-66, Lecture Notes in Math. 1745, Springer, Berlin, 2000.

[7] J. Bourgain, On the spectrum of lattice Schrödinger operators with deterministic potential, J. Anal. Math. 87 (2002), 37-75.

[8] J. Bourgain, On the spectrum of lattice Schrödinger operators with deterministic potential (II), J. Anal. Math. 88 (2002), 221-254.

[9] J. Bourgain, Estimates on Green's functions, localization and the quantum kicked rotor model, Ann. of Math. (2) 156-1 (2002), 249-294.

[10] J. Bourgain, M. Goldstein, W. Schlag, Anderson localization for Schrödinger operators on $\mathbb{Z}$ with potentials given by the skew-shift, Comm. Math. Phys. 220-3 (2001), 583-621.

[11] M. Brin, G. Stuck, Introduction to dynamical systems. Cambridge University Press, Cambridge, 2002. xii+240 pp.

[12] V. Chulaevsky, Y. Sinai, Anderson Localization for the I-D Discrete Schrödinger Operator with Two-Frequency Potential. Comm. Math, Phys. 125 (1989), 91-112.

[13] D. Damanik, H. Krüger, Almost Periodic Szegö Cocycles with Uniformly Positive Lyapunov Exponents. J. Approx. Theory 161:2, 813-818 (2009).

[14] A. Furman, On the multiplicative ergodic theorem for uniquely ergodic systems. Ann. Inst. H. Poincar Probab. Statist. 33:6 (1997), 797-815.

[15] F. Gesztesy, M. Zinchenko, Weyl-Titchmarsh theory for CMV operators associated with orthogonal polynomials on the unit circle. J. Approx. Th. 139 (2006), 172-213.

[16] F. Gesztesy, M. Zinchenko, A Borg-type theorem associated with orthogonal polynomials on the unit circle. J. Lond. Math. Soc. (2) 74 (2006), 757-777.

[17] M. Goldstein, W. Schlag, On resonances and the formation of gaps in the spectrum of quasi-periodic Schrödinger equations. Ann. of Math. (to appear).

[18] M. Goldstein, W. Schlag, On the formation of gaps in the spectrum of Schrödinger operators with quasi-periodic potentials. Spectral theory and mathematical physics: a Festschrift in honor of Barry Simon's 60th birthday, 539-563, Proc. Sympos. Pure Math., 76, Part 2, Amer. Math. Soc., Providence, RI, 2007.

[19] S. Jitomirskaya, B. Simon, Operators with singular continuous spectrum: III. Almost periodic Schrödinger operators. Commun. Math. Phys. 165 (1994), 201-205. 
[20] R. Killip, M. Stoiciu, Eigenvalue Statistics for CMV Matrices: From Poisson to Clock via Random Matrix Ensembles. Duke 146:3 (2009), 361-399.

[21] H. Krüger, A family of Schrödinger Operators whose spectrum is an interval. Comm. Math. Phys. 290:3 (2009), 935-939.

[22] H. Krüger, Probabilistic averages of Jacobi operators. Comm. Math. Phys. 295:3 (2010), 853-875.

[23] H. Krüger, Positive Lyapunov Exponent for Ergodic Schrödinger Operators. PhD Thesis, Rice University, April 2010.

[24] H. Krüger, On the spectrum of skew-shift Schrödinger operators, J. Funct. Anal. (to appear).

[25] Y. Last, B. Simon, The essential spectrum of Schrödinger, Jacobi, and CMV operators. J. d'Analyse Math. 98 (2006), 183-220.

[26] H. L. Montgomery, Ten lectures on the interface between analytic number theory and harmonic analysis, CBMS Regional Conference Series in Mathematics, 84. Published for the Conference Board of the Mathematical Sciences, Washington, DC; by the American Mathematical Society, Providence, RI, 1994. xiv+220 pp.

[27] T. van Ravenstein, The three gap theorem (Steinhaus conjecture). J. Austral. Math. Soc. Ser. A 45:3 (1988), 360-370.

[28] B. Simon, Regularity and the Cesáro-Nevai class, J. Approx. Theory 156 (2009), 142-153.

[29] B. Simon, Orthogonal Polynomials on the Unit Circle. Part 1. Classical Theory, Colloquium Publications, 54, American Mathematical Society, Providence (2005)

[30] B. Simon, Orthogonal Polynomials on the Unit Circle. Part 2. Spectral Theory, Colloquium Publications, 54, American Mathematical Society, Providence (2005)

[31] B. Simon, CMV matrices: Five years after. J. Comput. Appl. Math. 208 (2007), 120-154.

[32] B. Simon, OPUC on one foot. Bull. Amer. Math. Soc. 42 (2005), 431-460.

[33] B. Simon, Szegö's Theorem and its Descendants.

[34] M. Stoiciu, The statistical distribution of the zeros of random paraorthogonal polynomials on the unit circle. J. Approx. Theory 39 (2006), 29-64.

Mathematics 253-37, Caltech, Pasadena, CA 91125

E-mail address: helge@caltech.edu

$U R L:$ http://www.its.caltech.edu/ ${ }^{\sim h e l g e / ~}$ 